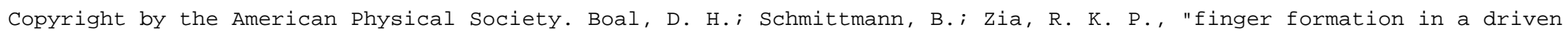
diffusive system," Phys. Rev. A 43, 5214 (1991); DOI: http://dx.doi.org/10.1103/PhysRevA.43.5214

\title{
Finger formation in a driven diffusive system
}

\author{
D. H. Boal \\ Department of Physics, Simon Fraser University, Burnaby, British Columbia, Canada V5A 1S6 \\ B. Schmittmann \\ Center for Stochastic Processes in Science and Engineering and Department of Physics, \\ Virginia Polytechnic Institute and State University, Blacksburg, Virginia 24061 \\ and Institut für Theoretische Physik, Universität Düsseldorf, D-4000 Düsseldorf, Germany \\ R. K. P. Zia \\ Center for Stochastic Processes in Science and Engineering and Department of Physics, Virginia Polytechnic Institute \\ and State University, Blacksburg, Virginia 24061
}

(Received 23 January 1991)

\begin{abstract}
A driven diffusive lattice gas is studied in a rectangular geometry: particles are fed in at one side and extracted at the other, after being swept through the system by a uniform driving field. Being periodic in the transverse direction, the lattice lies on the surface of a cylinder. The resulting nonequilibrium steady state depends strongly on this choice of boundary conditions. Both Monte Carlo and analytic techniques are employed to investigate the structure of typical configurations, the density profile, the steady-state current, and the nearest-neighbor correlations. As the temperature is lowered in a finite system, the simulations indicate a crossover from a disordered to an ordered state that is characterized by a backgammonlike pattern of alternating high- and low-density regions ("fingers"). For fixed strengths of the field and interparticle attraction, the average number of fingers is controlled by the ratio of the transverse to the longitudinal system size. Whether the crossover corresponds to an actual phase transition, where typical thermodynamic observables become singular, remains to be determined.
\end{abstract}

\section{INTRODUCTION}

Our understanding of nonequilibrium phenomena in even the simplest interacting many-particle systems is still very far from complete. Over the past years, considerable effort ${ }^{1-9}$ has been devoted to the study of various nonequilibrium steady states in stochastic lattice gases. In these simple model systems, particles hop to empty nearest-neighbor sites under the influence of a shortranged, Ising-like interaction between the particles, and a simulated thermal bath. Nonequilibrium steady states are easily established, and maintained, by suitable choices of boundary conditions or driving forces.

Our interest in this type of model system is threefold. First, it offers wide-ranging applications to real materials, such as binary mixtures, ${ }^{10}$ fast ionic conductors, ${ }^{11}$ and hydrogen in metals. ${ }^{12}$ Second, its static equilibrium properties are very well understood: they are described by the canonical, or the grand-canonical ensemble, depending on whether the system is coupled to a particle reservoir or not. Third, its dynamics around equilibrium can be cast in terms of well-established Langevin equations for the slow variables, ${ }^{13}$ which are accessible by methods of renormalized field theory. ${ }^{14}$ In particular, the equilibrium systems are known to undergo a second-order transition, whose static universality class is that of the Ising model, for spatial dimensions below 4. The dynamical universal behavior is described by model $B$ if the particle-particle interactions are attractive, and by model $A$ in the repulsive case. ${ }^{8}$ Thus, our analysis starts from well-explored ground.

In order to drive the system out of equilibrium, two major scenarios have been considered so far. In the first one, ${ }^{1}$ a nonequilibrium state is established by imposing a density, or chemical potential, gradient, in a rectangular geometry: particles are inserted at one edge, and extracted at the opposite edge, according to specified rates, thus fixing the boundary densities. Since the external driving forces act only at the surface, the steady-state current vanishes in the thermodynamic limit, so that the dominant characteristics of the system are equilibriumlike. This does not imply, however, that nonequilibrium effects occur only in a boundary layer. Rather, these effects must be uniformly present in the bulk, but with vanishing amplitudes.

In contrast, the second scenario ${ }^{2,3}$ involves external drives acting in the bulk and provides nonequilibrium effects in the thermodynamic limit. Typically, the driving field is chosen to point along one of the lattice axes and introduces an anisotropic bias into the hopping rates: jumps along (against) the field direction are favored (suppressed) while transverse jumps remain unaffected. One might think of the particles as carrying "electric" charge, driven by an external "electric" field. With fully periodic boundary conditions, the system settles into a steady state characterized by a nonzero current.

(c) 1991 The American Physical Society 
In this paper, we analyze a third scenario, which is a combination of the two described above: we study the usual driven diffusive lattice gas in a rectangular geometry, with different densities imposed at the bottom and the top edge. For simplicity, we consider only the case in which the bottom edge is always fully occupied while the top edge is completely empty, with the driving field directed from bottom to top. In the directions orthogonal to the field, we choose periodic boundary conditions. Also, our study is restricted to attractive particleparticle interactions.

It is to be expected that this choice of boundary conditions will generate a new type of nonequilibrium steady state, different from both the purely field and purely chemically driven systems. Our goal is to explore, and understand, the phenomenology of the associated steadystate configurations, as temperature and field strength are varied.

This paper is organized as follows. In Sec. II, we describe our model in more detail, and discuss its realization for Monte Carlo studies. In Sec. III, we present the simulation results. Our analytic and intuitive understanding, together with some open questions, are given in Sec. IV. Section V contains concluding remarks.

\section{MODEL}

We start from the familiar Ising lattice gas, defined on a two-dimensional square lattice. Each lattice site can be either vacant or occupied by a single particle, which models a hard-core exclusion. Different configurations are labeled by a set of occupation variables $\left\{n_{x y}\right\}$, with $n_{x y}=0$ or 1 , depending on occupancy. The indices $x=1,2, \ldots, W$ and $y=1,2, \ldots, L$ refer to the $x$ and the $y$ axis, respectively. The energy of a specific configuration is given by the "Hamiltonian"

$$
\beta \mathcal{H}=-J \sum n_{x y} n_{x^{\prime} y^{\prime}}-E \sum_{x, y} y n_{x y}
$$

with the first summation over nearest-neighbor pairs only. The exchange constant $J$ is positive, corresponding to attraction between particles (i.e., a "ferromagnetic" interaction, if the lattice gas is phrased in terms of Ising spins). The driving field $E$ points along the $y$ axis and specifies the "parallel" direction. The inverse temperature of the heat bath is denoted by $\beta$.

Next, we need to specify the transition rates according to which a given configuration evolves into a new one. For simplicity, we only allow for particle jumps to nearest-neighbor empty sites. We choose the usual Metropolis rates, ${ }^{15}$ with the following modification in order to incorporate the boundary densities: Particles diffuse into the system from row $y=0$, which is always fully occupied, and leave through row $y=L+1$, which is always completely empty. At the beginning of each sweep through the lattice, an attempt is made to fill each of the empty sites in row 1 by moving a particle from row 0 . The associated change in energy is calculated without reference to row 0 . Also, when particles leave through row $L$, row $L+1$ does not enter into the energy.

These boundary conditions, unlike toroidal ones, ${ }^{2}$ al- low us to write a "Hamiltonian" like (2.1). However, we stress that it merely provides a convenient way to express transition rates. Most importantly, $\exp (-\beta \mathcal{H})$ must not be confused with the steady-state distribution, which is determined entirely by the dynamics.

Simulations have been performed for system sizes $100 \leq L \leq 200$ and $W=50$ or 100 . Both the coupling constant $J$ and the driving field $E$ are measured in units of $\beta$. Thus, small values of $J$ correspond to high temperatures, and vice versa. We investigated $0 \leq J \leq 2.5$ and $1 \leq E \leq 5$.

A sweep across the $N$ particles occupying the lattice is defined as a total of $N$ trial moves on the particle ensemble. The particles are chosen randomly, so that in any given sweep some particles may be moved more than once and some not at all. We have also implemented an algorithm in which particles are moved sequentially by label and found that the calling sequence does not affect the results.

At the beginning of each run, the lattice is completely empty. It gradually fills up, as particles diffuse from the boundary into the bulk. Eventually, the particle density approaches its steady-state value of $\frac{1}{2}$, which may be expected since the dynamics is symmetric under CP transformations (defined by particle $\Longleftrightarrow$ hole and $y \Longleftarrow-y$ ). The time it takes to reach steady state, i.e., the "filling time," increases with $J$ and decreases with $E$. In Fig. 1, we show three sample results of the filling of a lattice with $L=200$ (the largest $L$ used in our simulations). For ease of comparison, the field has been fixed at $E=5$, while the exchange constant $J$ is varied. One can see that

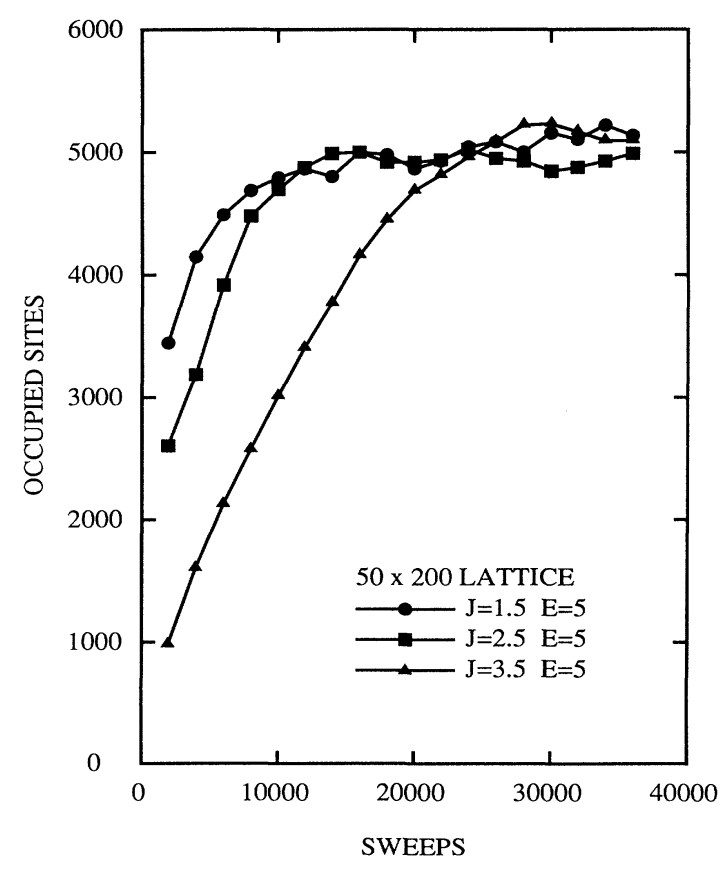

FIG. 1. Number of particles in a $50 \times 200$ lattice as a function of the number of sweeps. Initially the lattice is empty. Results are shown for $J=1.5,2.5$, and 3.5 and fixed $E=5$. (See text for the definition of a sweep.) 
the filling time for $J=3.5$ is about three times larger than that for $J=1.5$. Similarly, if $J$ is fixed at 2.5 , it takes several times longer to fill the lattice with $E=1$ than it does with $E=5$.

The filling times are of importance to the simulations because we wish to study steady-state phenomena. Barring unexpected long-time tails, we may assume that the system is in steady state by allowing it to evolve for Monte Carlo times long compared to the filling time. From Fig. 1, this implies that systems must evolve for at least 100000 Monte Carlo steps. Another quantity providing a time scale is the current, defined as the number of particles leaving the lattice per sweep, divided by $W$. Typical currents may be down to 0.005 , which implies a transit time of 20000 sweeps for a half-occupied lattice of length $L=200$. Hence, for most parameter combinations, we choose to run the simulation for 200000 sweeps. For $J=1.5$ and 2.0 at $E=1$, the samples are taken after 400000 and 800000 sweeps, respectively, because of the long transit times. The ensemble averages discussed in the following sections are made with ten configurations, each generated from a different initialization run for 200000 sweeps or more.

\section{SIMULATION RESULTS}

We begin this section with a qualitative discussion of typical configurations, for different system sizes, exchange constants, and fields. In general, at fixed $E$ we find that the system changes from a disordered state for $J \lesssim 1$ to an ordered state for $J \gtrsim 2$. The nature of the ordering is illustrated in Fig. 2, which shows steady-state configurations in a $50 \times 200$ lattice as observed after 200000 sweeps. The field is fixed at $E=5$, while the exchange constant takes the values $J=1.0,1.5$, and 1.7. At $J=1.0$, the steady state is disordered, but around $J=1.5$, the system is beginning to order, and we can see three "fingers" forming. For large values of $J$, these fingers become very sharp, thus reminding us of the pattern on a backgammon board.

Clearly one needs to test whether the finger formation is a true steady-state effect rather than a transient, or metastable, phenomenon. To do so, we have run simulations at strong attraction $J=2.5$ and $E=5$, starting from an initial configuration consisting of a half-filled lattice occupied by a single finger. For both $100 \times 100$ and $100 \times 200$ lattices, after 50000 sweeps, this finger breaks up into the same fingering pattern as obtained from an initially empty lattice. Hence we may conclude that the finger pattern corresponds to a generic steady-state feature.

If both $J$ and $E$ are kept fixed, the number of fingers decreases with the ratio $W / L$, as demonstrated in Fig. 3 . In this sequence, $W$ is held constant at 100 , while $L$ increases from 100 via 140 to 200 . The number of fingers, $N_{f}$, is observed to decrease from eight to six and then down to four. For fixed $J, E$, and $W / L$ there are small fluctuations in the number of fingers: the latter may differ by one between different configurations. However, the average number of fingers $\left\langle N_{f}\right\rangle$, formed in a sequence of runs starting from an empty lattice, varies

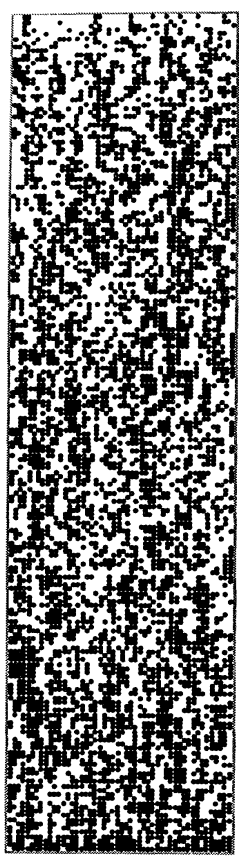

(a)

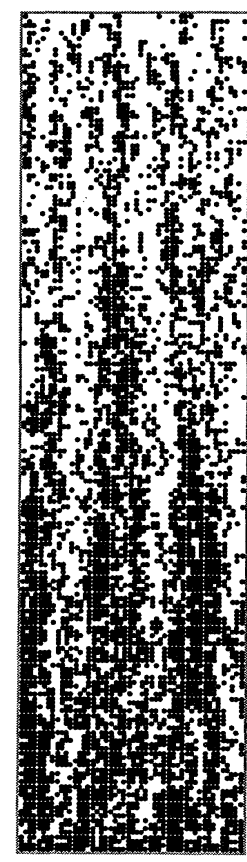

(b)

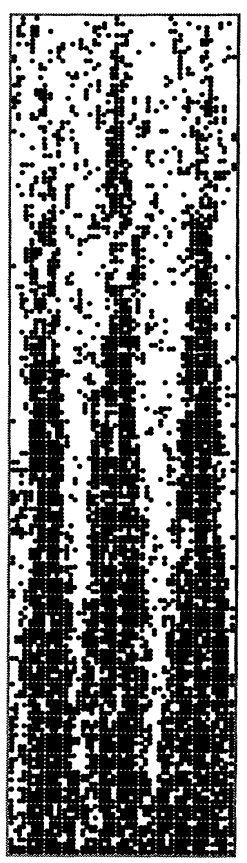

(c)
FIG. 2. Typical configurations on a $50 \times 200$ lattice, taken after 200000 sweeps, with $J=$ (a) 1.0 , (b) 1.5 , and (c) 1.7 , and $E=5$.

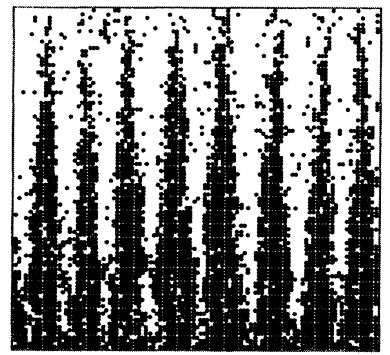

(a)

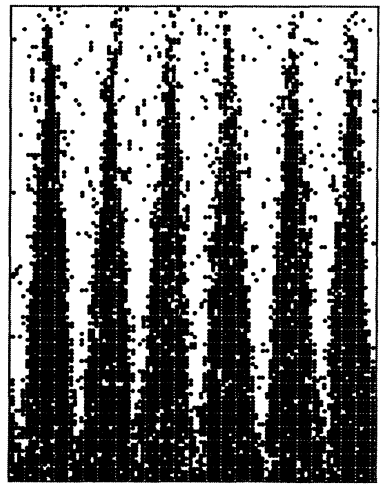

(b)

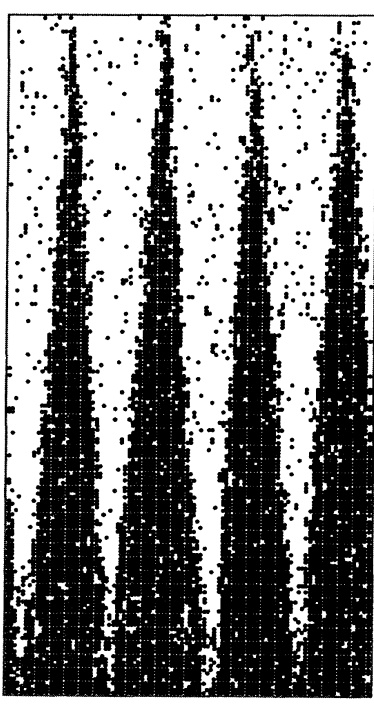

(c)
FIG. 3. Typical configurations in the low-temperature phase, with $J=2.5$, and $E=5$, taken after 200000 sweeps. Lattices with fixed $W$ and varying $L$ are chosen: (a) $100 \times 100$, (b) $100 \times 140$, and (c) $100 \times 200$. 
smoothly with $W / L$, as shown in Fig. 4 . The angle formed by the individual interfaces with the parallel direction, defined via $\tan \theta=\left\langle W / 2 L N_{f}\right\rangle$, is relatively constant as a function of $W / L$. We also detect a small, systematic finite-size effect, due to the length of the fingers not being exactly $L$. For the range of parameters in Fig. 4, a linear form $(a+b / L)$ fits $\tan \theta$ quite well, with $a=0.038 \pm 0.004$.

It is interesting to compare our results for this angle with data obtained for shifted periodic boundary conditions along the field direction. ${ }^{7}$ In the latter case, typical low-temperature configurations at small shifts are single strips that break up into multiple strips as the shift is increased. As a check, we repeated simulations of this model, also for $E=5$ and $J=2.5$, on a $100 \times 100$ lattice. We find that the (critical) angle for multiple-strip formation is roughly twice as large as the fingering angle. Since the physics of the bulk ordering associated with these angles is quite distinct, quantitative comparisons will not be given.

Finally, note that, at fixed $W / L$ and $E$, the number of fingers decreases with increasing $J$. This behavior might be expected from a system with larger $J$ to favor smaller total interface length.

Next, we turn to a discussion of the density profile,

$$
\rho(y) \equiv \sum_{x}\left\langle n_{x y}\right\rangle / W
$$

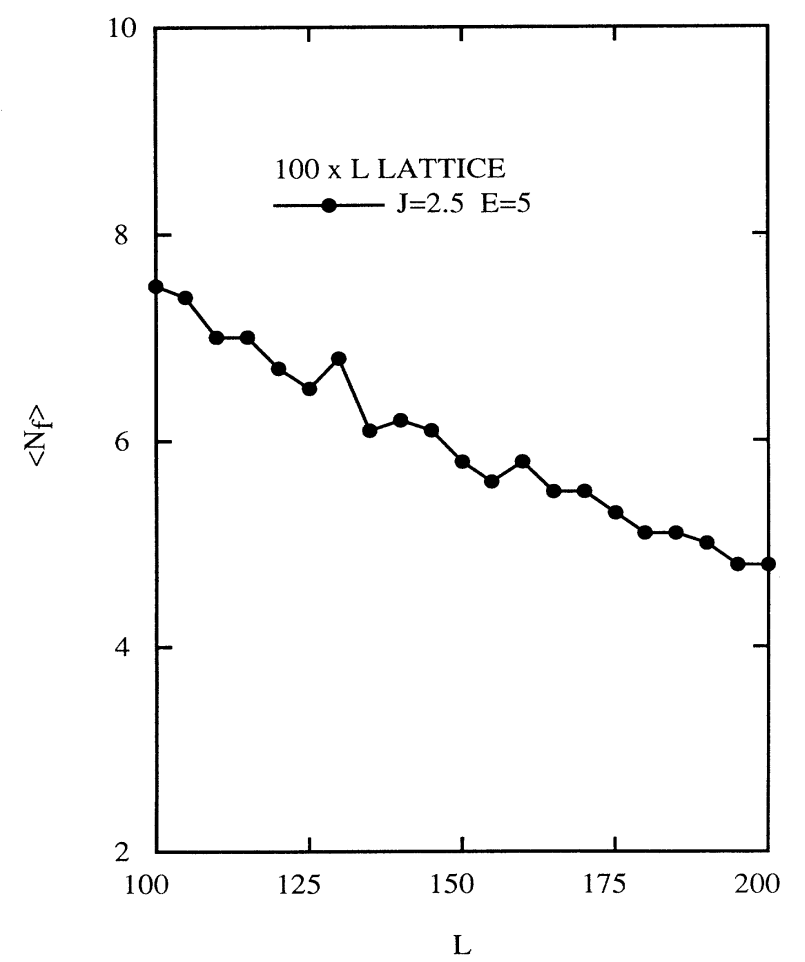

FIG. 4. Average number of fingers, $N_{f}$, as a function of $L$ for a $100 \times L$ lattice, with $J=2.5$ and $E=5$. The average is taken over ten configurations, each being the result of different initializations run for 200000 sweeps.
Figure 5 indicates that the crossover from random occupation to fingering is accompanied by a change in the shape of $\rho(y)$ : For $J=1, \rho(y)$ varies strongly at the boundaries, while remaining fairly constant, with a small negative slope, through the rest of the lattice. As $J$ increases, the profile gradually crosses over to a linearly decreasing function of $y$. The latter corresponds to a perfect "backgammon" pattern of fingers. Figure 5 also shows that the density profile is antisymmetric around $y=L / 2$, apart from an overall average density $\frac{1}{2}$, as expected from $\mathbf{C P}$ symmetry.

The average number $C_{\mathrm{NN}}$ of nearest-neighbor bonds per occupied site is shown in Fig. 6. For a system of free particles $(J=0)$, the configurations are completely random, corresponding to an average of two neighbors per particle. As $J$ becomes larger, $C_{\mathrm{NN}}$ increases, due to the stronger attraction between the particles. Note that the total number of occupied sites does not change appreciably, due to CP symmetry. Since $C_{\mathrm{NN}}$ and the average "internal" energy per site differ only by a (temperatureindependent) factor, $C_{\mathrm{NN}}$ can be used to extract the specific heat, defined as the temperature derivative of $-J \sum\left\langle n_{x y} n_{x^{\prime} y^{\prime}}\right\rangle$. From Fig. 6, we might expect a peak in the specific heat, located around $J=1.7$, for $E=5$, and possibly at a slightly smaller value of $J$ for $E=3$ and 1 . However, more data and detailed finite-size analysis are needed before we can be certain of the existence or absence of a true singularity. Only then could a reliable picture of a phase transition emerge.

Finally, we comment on $j$, the average current through

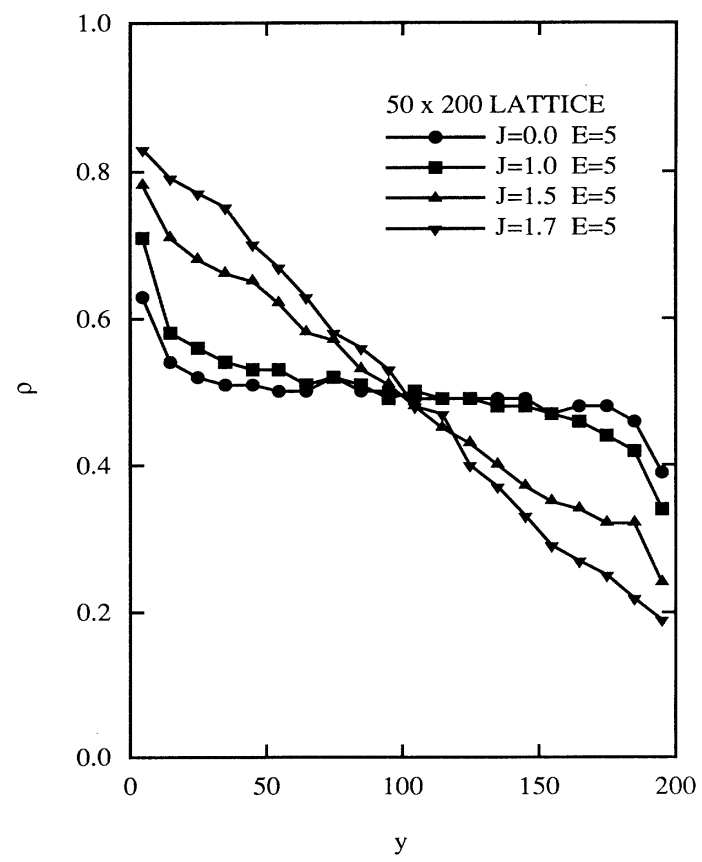

FIG. 5. Density profile $\rho$ as a function of $y$, the distance from the row of particle entry into the lattice, for four values of $J=0$, $1.0,1.5$, and 1.7 , and fixed $E=5$. The averaging procedure is the same as in Fig. 4. 


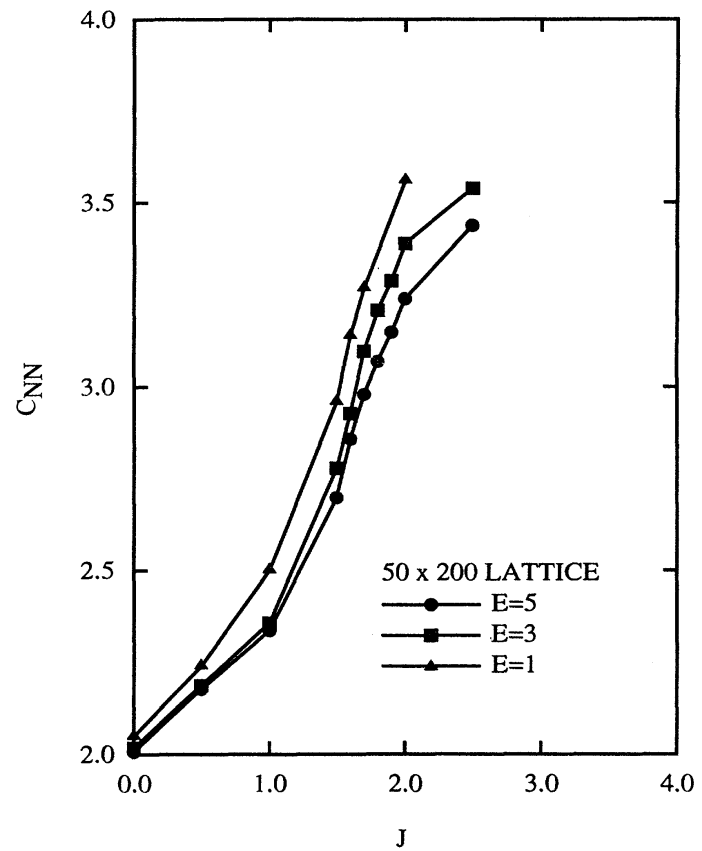

FIG. 6. Average number of nearest neighbors per particle, $C_{\mathrm{NN}}$, on a $50 \times 200$ lattice shown as a function of $J$, for three values of $E=1,3$, and 5 .

the lattice. It can be measured very simply as $1 / W$ times the number of particles that leave the system through row $L+1$ during each sweep. Figure 7 shows the behavior of the current at fixed lattice parameters $W$ and $L$ : The current increases with $E$ and decreases with $J$. At

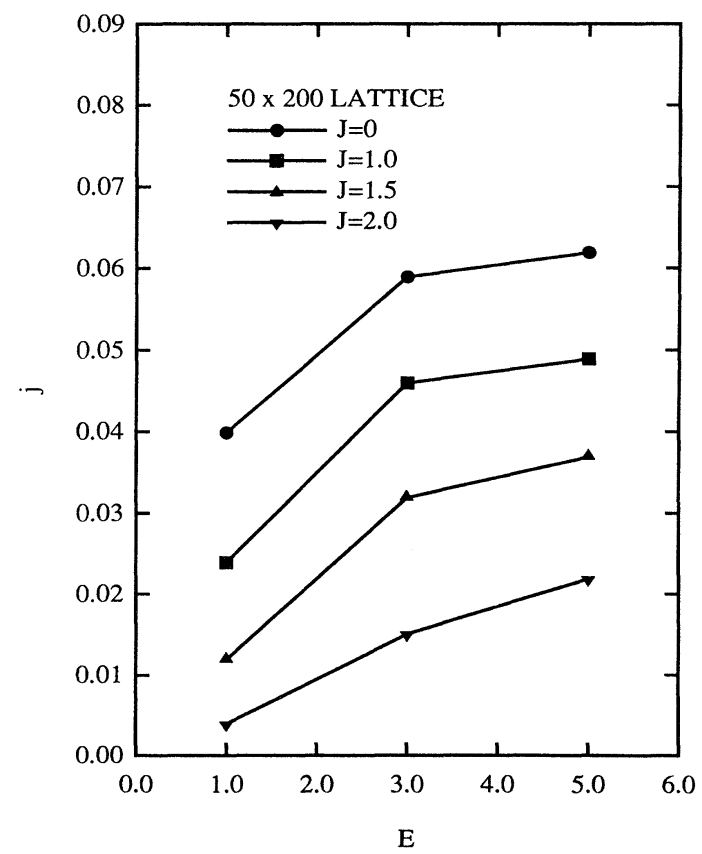

FIG. 7. Dependence of $j$, the particle current, on $J$ and $E$ for fixed lattice size. The current is defined as the number of particles leaving the top row per sweep, divided by $W$, the number of sites in a row.

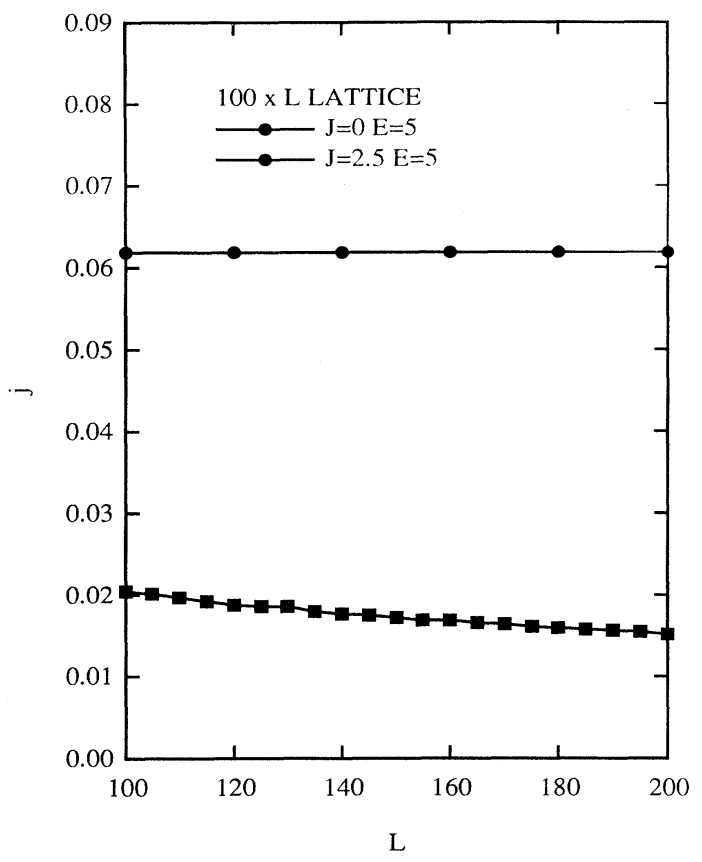

FIG. 8. Lattice size ( $L$ ) dependence of $j$, the current at fixed $J, E=5$, and $W=100$. Two cases are shown: $J=0$ (no fingers) and $J=2.5$ (fingers).

fixed $J$, it will saturate for sufficiently large fields, similar to the behavior of the standard driven diffusive systems (DDS) (Refs. 2 and 3) with periodic boundary conditions (PBC).

The $L$ dependence of the current is of some interest. Unlike a model without drive, ${ }^{1}$ our current should be finite in the $L \rightarrow \infty$ limit. We illustrate this dependence in Fig. 8, using samples with fixed width $(W=100)$ and field $(E=5)$. The two values of the exchange constant chosen correspond to disordered $(J=0)$ and ordered phases $(J=2.5)$. For $J=0$, the current displays little or no lattice size dependence for the range of $L$ shown. In contrast, at $J=2.5$, the current shows a weak dependence on $L$, decreasing from 0.021 to 0.015 . This behavior might be related to the $L$ dependence of the number of fingers, which drops from 7.5 to 4.8 over the same range of $L$. For later reference, we wish to compare these results to the PBC model. For $J=0$, we find the currents to be the same for both cases. However, for $J=2.5$, our model carries much more current than the PBC model. We will return to these observations in the following section.

\section{ANALYTIC RESULTS AND DISCUSSION}

In this section, we present our analytic understanding of the steady-state configurations. To begin with, we need an analytic formulation of the simulation dynamics. While the latter can, in principle, be cast in terms of a master equation with suitably chosen transition rates, this description is not very successful in practice. Very rarely is it possible to determine the exact steady-state distribu- 
tion in this way. Instead, we proceed along the route proven extremely useful for predicting universal behavior in critical dynamics. ${ }^{13}$ The starting point is a set of Langevin equations of motion for macroscopic, slowly varying degrees of freedom, with noise terms modeling the effects of the fast degrees of freedom. Such equations are sometimes derived. ${ }^{16}$ However, more often, they are postulated, taking into account the symmetries and conservation laws of the microscopic dynamics.

In this spirit, we analyze our model in terms of an equation of motion for the local density $\rho(r, t)$. First, we discuss the dynamics for the noninteracting gas $(J=0)$, since this case can be exactly solved. Next, we establish the equation of motion for finite $J$, governing the dynamics of both the ordered and disordered phase. In a small$J$ expansion, we compute the first-order corrections to the "free" solution. The results agree well with the simulations of the density profile. The analysis of the lowtemperature phase is much more complicated since the profile should be inhomogeneous in both spatial variables. Such analytic solutions to nonlinear partial differential equations are hard to find, so that we are restricted to a discussion of the mechanism that we believe to be responsible for the formation of fingers.

\section{A. The noninteracting case}

At $J=0$, the usual driven system reduces to a model of biased hopping, or diffusion:

$$
\partial_{t} \rho(r, t)=D_{\perp} \nabla_{\perp}^{2} \rho+D_{\|} \partial^{2} \rho-E \partial \rho(1-\rho) .
$$

Here, $\nabla_{\perp}(\partial)$ denotes the gradient with respect to the coordinates transverse (parallel) to the field. The corresponding diffusion coefficients are denoted by $D_{\perp}$ and $D_{\|}$, while $E \neq 0$ introduces a bias in the parallel direction. To account for the particle sources and sinks at the edges of our system, this equation must be supplemented with a boundary condition on $\rho(r, t)$ : we require $\rho(x, y=0, t)$ $=1$ and $\rho(x, y=L, t)=0$ for all $y$ and $t$. The stochastic version of this equation, in the absence of boundary conditions, has been studied, ${ }^{17}$ with the focus on anomalous long-time, long-wavelength behavior, in dimensions less than 2.

The origin of Eq. (4.1) can be understood as follows. First, in the absence of the bias, the usual diffusion equation can be rewritten as $\partial_{t} \rho=\nabla \cdot D(\rho) \nabla\left(\delta H_{0} / \delta \rho\right)$, where $\boldsymbol{\nabla}$ is the full gradient operator. Here we must consider a density-dependent transport coefficient $D(\rho)$ and a "free" Hamiltonian $\mathscr{H}_{0}$, which comes from the entropy of a system of $N \rho$ particles distributed over $N$ sites. To lowest order in $\rho, D(\rho)=D_{0} \rho(1-\rho)$, to account for the fact that the (local) current is zero in an environment that is either fully occupied or empty. This effect is clearly crucial at both boundaries of the system. Second, a nonzero bias induces an extra contribution to the diffusion current. We write it in the form $\mathbf{j}=\sigma(\rho) \mathbf{E}$, where $\mathbf{E}$ is a measure for the bias, and the conductivity $\sigma$ depends on the (local) density via $\sigma(\rho)=\sigma_{0} \rho(1-\rho)$, in analogy to $D(\rho)$. The anisotropy of the bias then generates effective anisotropic diffusion constants $D_{\|}$and $D_{\perp}$, while $\sigma_{0}$ is absorbed into $E$. We caution that the $E$ here should not be confused with the microscopic $E$ that appears in the preceding sections. In particular, even if the microscopic $E$ is infinite, the total current saturates at a finite value, so that $E$ here is also finite.

In the transverse subspace, the system is translation invariant, due to the periodic boundary conditions. We therefore seek a stationary solution to Eq. (4.1) that depends on the parallel coordinate $y$ only. After some trivial rescalings, the (stationary) local "magnetization," $\phi(y) \equiv 2 \rho(y)-1$, obeys the equation

$$
0=\partial^{2} \phi-\partial E\left(1-\phi^{2}\right)
$$

with the boundary conditions $\phi( \pm L / 2)=\mp 1$. Note that we have shifted the origin $y=0$ to the center of the system for convenience. For vanishing $E$, the solution is the well-known $\phi=-2 y / L$. For $E \neq 0$, the density profile can no longer be linear. Instead, we find

$$
\phi_{0}(z)=-A \tan (\mu z),
$$

where

$$
A \equiv \frac{2 \mu}{L E} \quad \text { and } \quad z \equiv \frac{2 y}{L} .
$$

The subscript on $\phi$ is a remainder of $J=0$. Imposing the boundary conditions gives an equation for fixing the unknown $\mu$ :

$$
\mu \tan \mu=\frac{1}{2} L E .
$$

Note that the profile (4.3) has a curvature opposite to the more familiar tanh-like ones, which appear in systems ${ }^{1}$ with $J>0$ but $E=0$. This difference is expected, because $J$ tends to gather particles at, while $E$ drives them away from, the high-density edge. Since the effect of $J$ on a totally free system is opposite to that of $E$, it is natural to ask whether they might cancel each other and lead to a linear density profile for finite $J$ and $E$. As we will see, this conjecture is confirmed to first order, in a perturbative treatment of $\dot{J}$.

In this theory, $1 / E$ provides a length scale and $\phi_{0}(z)$ depends on the system size and the field strength through the combination $\frac{1}{2} L E$ only. Thus, the zero-drive limit and the thermodynamic limit are not interchangeable. If we take the former first, $\mu$ will vanish according to $\mu^{2}=\frac{1}{2} L E\left(1-\frac{1}{2} L E / 3\right)+O\left((L E)^{3}\right)$, so that

$$
\phi_{0}(z)=-z+\frac{1}{2} L E\left(z-z^{3}\right) / 3+O\left(z^{5},(L E)^{2}\right) .
$$

The linear profile is recovered at $E=0$ while the firstorder term provides curvature. On the other hand, if we take $L \rightarrow \infty$ first, we have $\mu \approx \pi / 2$. In this limit, $\phi_{0}$ is essentially zero (with vanishing slope $\approx-\pi^{2} / E L^{2}$ at $y=0$ ), except near the boundaries. This behavior agrees well with that in the simulations (cf. Fig. 5). Keeping in mind that we are considering the disordered phase, these features are expected since the effects of the sources and sinks on the bulk should vanish in the thermodynamic limit. The sharp rise in $\phi_{0}$ near either edge is reminiscent of a boundary layer. However, our profile does not decay exponentially into the bulk. In the thermodynamic limit, it decays inversely with distance. For example, if $\xi$ is the 
distance from the lower edge, $\phi_{0}=1 /(1+\xi E)$. Such a slow decay might be related to the slow power-law decays of correlation functions observed in DDS. ${ }^{5}$

The steady-state current through the system is the first integral of Eq. (4.2), given by $j_{0}=E\left(1+A^{2}\right)$. It also shows the crossover from one limit to the other. For $L \rightarrow \infty$, we have simply $j_{0}=E$, independent of $L$. On the other hand, when $E$ vanishes we are left with $j_{0}=2 / L$, as expected.

\section{B. The disordered phase}

Next, we turn to the effect of interparticle interactions and study $J>0$. In the absence of the driving force, the equation of motion should still be well modeled by $\partial_{t} \rho=\nabla \cdot D(\rho) \nabla(\delta \mathcal{H} / \delta \rho)$, with the same transport coefficient $D(\rho)$. But now, $\mathscr{H}$ should include interaction terms, in addition to the free $\mathcal{H}_{0}$. These terms may be obtained in the usual way by taking the continuum limit of the microscopic $-J \sum n_{x y} n_{x^{\prime} y^{\prime}}$ and coarse graining $n_{x y}$ to $\rho(x, y)$. We keep only the lowest-order terms and recognize that they would provide an effective Hamiltonian, appropriate for the mesoscopic description. Thus we write $-K \rho\left(1+a^{2} \nabla^{2}\right) \rho$, where $a$ is essentially the lattice spacing and $K$ denotes an effective coupling, vanishing when $J \rightarrow 0$. We remark that the resultant equation of motion, to lowest order in $\phi$ and $\nabla$, is just $\partial_{t} \phi(r, t) \propto \nabla \cdot\left[(1-K) \nabla \phi+K \phi^{2} \nabla \phi-K a^{2} \nabla \nabla^{2} \phi\right], \quad$ which successfully describes the critical dynamics of a conserved order parameter. ${ }^{13,14}$ Finally, including the current due to $E$, i.e., $E\left(1-\phi^{2}\right)$, should induce anisotropic transport coefficients as in (4.1).

If we add a conserved noise term to the right-hand side of such an equation, it becomes the basis for the fieldtheory analysis of critical behavior in the usual DDS. ${ }^{4}$ Unlike that case, we are now interested in the influence of specific boundary densities on the structure of steady states. Thus, we propose the same equation of motion (all anisotropies suppressed, for simplicity),

$\partial_{t} \phi(r, t)=\nabla \cdot\left[D \nabla \phi+g \phi^{2} \nabla \phi-\kappa \nabla \nabla^{2} \phi-\mathbf{E}\left(1-\phi^{2}\right)\right]$,

with, however, a different boundary condition, i.e.,

$$
\phi( \pm L / 2)=\mp 1 \text {. }
$$

We believe that these equations contain all the essential features to provide an adequate phenomenological description of our system, in the same spirit that a Landau-Ginzburg Hamiltonian represents the Ising system. Thus $(4.7 \mathrm{a})$ and $(4.7 \mathrm{~b})$ could be regarded as a meanfield theory, with fluctuations (and noise) ignored, valid for both high and low temperatures. We stress that the parameters here are (in principle known) functions of the microscopic $J, E$, and $T$. As in the Landau-Ginzburg theory, the disordered and ordered phases are modeled by positive and negative signs of the (transverse) coefficient $D$. Thus, in the crudest approximation, the transition occurs for $K=1$.

In the disordered phase, we may again assume homogeneity in the transverse subspace. Even then, we are un- able to find analytic stationary solutions to (4.7a) and (4.7b). However, we may consider small $J$ (and so, $K$ ), treated as perturbations to the free system, in analogy to high-temperature expansions for equilibrium systems. Using the continuum version, we may also neglect the $K a^{2} \nabla \nabla^{2} \phi$ term compared to $K \nabla \phi$, in this (far above $T_{c}$ ) regime. Thus, we seek solutions to

$$
0=\partial\left[\partial \phi-E\left(1-\phi^{2}\right)-K\left(1-\phi^{2}\right) \partial \phi\right]
$$

in the form of small $K$ expansions around $\phi_{0}$. Though the first-order correction is easily found by quadrature, its lengthy form offers little transparent insight and will not be explicitly quoted here. Instead we give only the change in the current,

$$
\delta j_{0} / j_{0}=-2 K A^{2}\left(\frac{1}{2} j_{0} L-1\right) /\left(\frac{1}{2} j_{0} L+1\right) .
$$

The combination $\frac{1}{2} j_{0} L$ is actually the ratio of $j_{0}$ to the current in an $E=0$ system, so that it is always greater than unity. Thus $\delta j_{0}$ is always negative, agreeing with intuitive assessments that attractive interactions reduce the current. More accurate data will be needed to check (4.9) quantitatively.

This perturbative solution provides an answer to a question raised above, i.e., do the opposing effects of $J$ and $E$ on the curvature of $\phi(y)$ cancel? It is clear that a single parameter like $K$ cannot cancel every effect of $E$ by changing (4.3) back to a linear form. However, if $E$ is also small, so that its effects appear simply [cf. (4.5)], the cancellation is possible. Thus, the density profile, to first order in $K$ and $E$, takes the form

$$
\phi(z)=-z+\left(\frac{1}{2} L E-K\right)\left(z-z^{3}\right) / 3+O\left(z^{5},(L E)^{2}, K^{2}\right),
$$

which becomes linear for $K=\frac{1}{2} L E$.

This study of the disordered phase shows that we can expect the thermodynamic limit of the bulk properties of this model to be identical to those in a driven system with PBC. Nevertheless, there are interesting questions concerning the slow decay of the boundary effects yet to be investigated.

\section{The low-temperature regime}

Finally, we comment on the low-temperature phase of our model. First, we should expect the coexistence of regions of high and low density, based on the display of spontaneous symmetry breaking and long-range order in the usual DDS. ${ }^{2}$ Unfortunately, the long-range order makes it difficult to build an intuitive picture of how the bulk might be affected by the particle sources and sinks at the boundaries. On the other hand, to find fully inhomogeneous steady-state solutions to $(4.7 \mathrm{a})$ and $(4.7 \mathrm{~b})$, with a negative $D_{1}$, is far from easy. We intend to pursue numerical solutions, although they usually offer little physical insight into whether these equations indeed capture the essence of the system. Until we develop more powerful analytical tools, we can only present a qualitative understanding of mechanisms that might be responsible for the formation of the backgammonlike pattern. 
For low temperatures, the driven system with periodic boundary conditions orders into a single (say, highdensity) strip oriented parallel to the field. Multiple strips do form, but with time they merge into a single one. However, configurations with strips orthogonal to $E$ are highly unstable, especially for large $E$. In other words, the steady state is always homogeneous in $y$. At half filling, ordering appears by phase separation into a state inhomogeneous in $x$. Our boundary conditions, in contrast, force precisely the opposite distribution. Indeed, in systems without drive, ${ }^{1}$ the sources and sinks do select configurations phase separated in $y$. The competition between $E$ and the boundary conditions leads naturally to states inhomogeneous in both directions.

Given that $J$ is large, we may expect the system to minimize interfacial tension by having a single high-density region, separated from a similar low-density region. For equilibrium Ising systems, this expectation alone is enough to predict the pattern at low temperatures. For our system, the simplest geometrical pattern, which accounts for the competing forces aligning the interface with $x$ versus $y$, would be just a single finger, with interfaces making an angle $\arctan \left(\frac{1}{2} W L\right)$ with respect to the field direction. However, the field will destroy such configurations if the "tilt" angle is too large ${ }^{7}$ leading to a multifinger pattern. On the other hand, this angle cannot be arbitrarily small, since that would require many more fingers and much larger interface "area," a situation not favored by large $J$. Based on these arguments, we conclude that some nontrivial angle exists and the backgammon configuration emerges as the stable pattern in the low-temperature phase.

We end with some remarks on the current. In the steady state, it is proportional to the number of broken bonds (or particle-hole pairs), oriented along the field direction. In the continuum theory, this fact is modeled by the factor $\rho(1-\rho)$ in the transport coefficient. Thus, in the phase-separated regime of a system with PBC, the current is carried mainly in the interfacial region, where the number of broken bonds is maximal. With the introduction of particle sources and sinks, we effectively impose an extra density gradient on the system, so that the current should be larger than in the PBC case (at the same $J, T$, and $E$ ). Indeed, as reported above, the data show a substantial increase in the current. This may be traced to the tilting of the orientation of the interface, so that there are many more broken bonds in the field direction. When a clearer picture of the stationary state density emerges, we will arrive presumably at a quantitative understanding of the current.

\section{CONCLUDING REMARKS}

Our theoretical pursuits, by no means complete, lead instead to a host of open questions, some of which are intimately related to each other. We close by presenting a partial list.

(a) Is there a thermodynamic limit of the lowtemperature phase? Indeed, we may expect many stable phases, depending on how this limit is taken, i.e., $L \rightarrow \infty$ first, $W \rightarrow \infty$ first, or $L / W$ fixed.

(b) Will the boundary effects disappear inversely with distance for the low-temperature phase? If so, we should see a single strip parallel to $E$ when $L$ is sufficiently large. In that case, how do $E$ and $W$ control this crossover length? If not, and a single finger remains for any $L$, how does $\tan \theta$ cross over from being a constant to being proportional to $1 / L$ ?

(c) For most values of $L / W$, typical runs show one or another number of fingers. We may ask if our system displays true bistability or if the data reflect metastability typical of a first-order transition (in $L / W$ ). Since we have a nonequilibrium system, it is possible for the former to exist in finite regions of phase space. ${ }^{18}$ Work is in progress to clarify this question.

(d) Obviously, we should investigate systems of higher dimensions. Since geometric factors like $L / W$ seem to control the ordered phases, the possibilities seem even more limitless there.

In conclusion, we have investigated only a small portion of the phase space in what appears to be an extremely rich system. Many questions remain, ready for explorations by various techniques, e.g., Monte Carlo, numerical, and analytic. Finally, being a system with open boundary conditions, it should be much easier to realize in the laboratory. We look forward to experimental evidence for the phenomena we have discovered here.

\section{ACKNOWLEDGMENTS}

We acknowledge illuminating discussions with Professor R. Frindt. Two of us (B.S. and R.K.P.Z.) thank Professor M. Plischke and Professor M. Wortis for their hospitality and support at Simon Fraser University, where this work was started. This research is funded in part by Natural Sciences and Engineering Research Council of Canada, the Sonderforschungsbereich 237 of the Deutsche Forschungsgemeinschaft, and the (U.S.) National Science Foundation through the Division of Materials Research.
${ }^{1}$ G. E. Murch, Philos. Mag. A 41, 157 (1980); H. Spohn, J. Phys. A 16, 4275 (1983); J. V. Andersen and O. G. Mouritsen, Phys. Rev. Lett. 65, 440 (1990); H. C. Fogedby and A. Svane, Phys. Rev. B 42, 1056 (1990).

${ }^{2}$ S. Katz, J. L. Lebowitz, and H. Spohn, Phys. Rev. B 28, 1655 (1983); J. Stat. Phys. 34, 497 (1984). For a recent review, see Ref. 3.
${ }^{3}$ B. Schmittmann, Int. J. Mod. Phys. B 4, 2269 (1990).

${ }^{4}$ K.-t. Leung and J. L. Cardy, J. Stat. Phys. 44, 567 (1986); H. K. Janssen and B. Schmittmann, Z. Phys. B 64, 503 (1986); K. Gawedzki and A. Kupiainen, Nucl. Phys. B 269, 45 (1986).

${ }^{5}$ M. Q. Zhang, J.-S. Wang, J. L. Lebowitz, and J. L. Vallés, J. Stat. Phys. 52, 1461 (1988).

${ }^{6}$ K.-t. Leung, J. Stat. Phys. 50, 405 (1988); A. Hernandez- 
Machado, and D. Jasnow, Phys. Rev. A 37, 626 (1988); K.-t. Leung, K. K. Mon, J. L. Vallés, and R. K. P. Zia, Phys. Rev. Lett. 61, 1744 (1988).

${ }^{7}$ J. L. Vallés, K.-t. Leung, and R. K. P. Zia, J. Stat. Phys. 56, 43 (1989); K.-t. Leung, J. Stat. Phys. 61, 341 (1990).

${ }^{8}$ K.-t. Leung, B. Schmittmann, and R. K. P. Zia, Phys. Rev. Lett. 62, 1772 (1989).

${ }^{9}$ B. Schmittmann and R. K. P. Zia, Phys. Rev. Lett. 66, 357 (1991).

${ }^{10}$ See, e.g., R. K. Pathria, Statistical Mechanics (Pergamon, Oxford, 1972), p. 399.

${ }^{11}$ See, e.g., W. Dieterich, P. Fulde, and I. Peschel, Adv. Phys. 29, 527 (1980), and references therein.

${ }^{12}$ See, e.g., H. Wagner and H. Horner, Adv. Phys. 23, 587 (1974).

${ }^{13}$ B. I. Halperin, P. C. Hohenberg, and S.-k. Ma, Phys. Rev. B 10, 139 (1974). For a review, see P. C. Hohenberg and B. I.
Halperin, Rev. Mod. Phys. 49, 435 (1977).

${ }^{14}$ H. K. Janssen, Z. Phys. B 23, 377 (1976); R. Bausch, H. K. Janssen and H. Wagner, ibid. 24, 113 (1976); C. De Dominicis, J. Phys. (Paris) Colloq. 37, C1-247 (1976).

${ }^{15}$ N. Metropolis, A. W. Rosenbluth, M. M. Rosenbluth, A. H. Teller, and E. Teller, J. Chem. Phys. 21, 1087 (1953).

${ }^{16}$ M. Lax, Rev. Mod. Phys. 32, 25 (1960); H. Mori, Prog. Theor. Phys. 33, 423 (1965); P. C. Martin, in Many Body Physics, edited by C. De Witt and R. Balian (Gordon and Breach, New York, 1968); R. Zwanzig, in Statistical Mechanics, edited by S. A. Rice, K. F. Freed, and J. C. Light (University of Chicago Press, Chicago, 1972); N. G. van Kampen, Phys. Rep. 24C, 171 (1976).

${ }^{17}$ H. K. Janssen and B. Schmittmann, Z. Phys. B 64, 503 (1986).

${ }^{18}$ C. H. Bennett and G. Grinstein, Phys. Rev. Lett. 55, 657 (1985). 